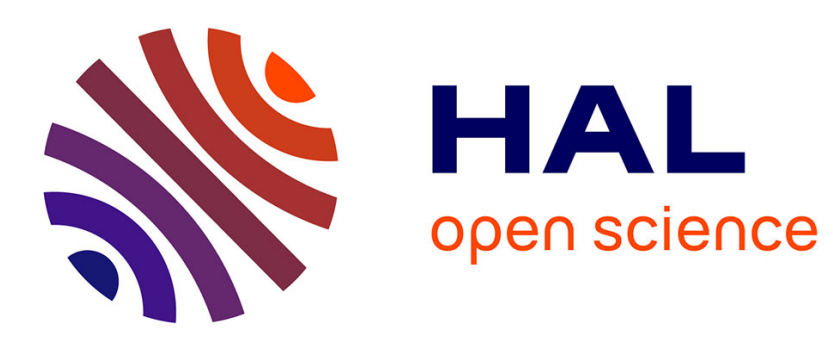

\title{
Deux problèmes pour une épistémologie sociale
}

Pierre Willaime

\section{To cite this version:}

Pierre Willaime. Deux problèmes pour une épistémologie sociale. Implications philosophiques, 2016. halshs-01280834

\section{HAL Id: halshs-01280834 \\ https://shs.hal.science/halshs-01280834}

Submitted on 1 Mar 2016

HAL is a multi-disciplinary open access archive for the deposit and dissemination of scientific research documents, whether they are published or not. The documents may come from teaching and research institutions in France or abroad, or from public or private research centers.
L'archive ouverte pluridisciplinaire HAL, est destinée au dépôt et à la diffusion de documents scientifiques de niveau recherche, publiés ou non, émanant des établissements d'enseignement et de recherche français ou étrangers, des laboratoires publics ou privés. 


\title{
Deux problèmes pour une épistémologie sociale
}

\author{
Pierre Willaime* \\ Laboratoire d'Histoire des Sciences et de Philosophie \\ Archives Henri Poincaré \\ UMR 7117 CNRS / Université de Lorraine
}

25 décembre 2015

\begin{abstract}
Résumé
Cet article présente brièvement deux problèmes qui peuvent survenir lors de la construction d'une épistémologie sociale. Le premier problème est une conséquence directe de l'adhésion au fiabilisme et touche donc l'épistémologie sociale telle que développée par Alvin Goldman (1999). Son utilisation du fiabilisme coupe la remontée de la chaîne des justifications et propose en définitive une évaluation des pratiques épistémiques qui ne dépend pas de leur mode de construction. L'analyse du cas du novice et des deux experts permet de s'en rendre compte. Une telle épistémologie sociale fiabiliste redéfinit les notions épistémiques classiques en termes naturalistes et se détache ainsi des débats de la philosophie de la connaissance contemporaine. Les problèmes soulevés par cette approche sont nombreux et motivent le développement d'une autre théorie de la justification pour fonder l'épistémologie sociale ; cette dernière refuserait la scission implicite entre épistémologie traditionnelle et sociale.

Le second problème touche l'épistémologie des vertus considérée comme une théorie candidate à la formation de cette nouvelle épistémologie sociale. Si l'épistémologie des vertus offre un cadre adéquat pour la description des pratiques épistémiques, elle semble trop imprécise pour être un guide efficace à l'action et contribuer à l'identification des bonnes pratiques épistémiques comme le veut l'épistémologie sociale. Une solution possible pourrait être de l'articuler avec des études de cas.
\end{abstract}

L'épistémologie sociale poursuit deux objectifs principaux; le premier d'entre eux est le développement d'une théorie de la connaissance (et de la justification) qui ne soit pas uniquement centrée sur les processus cognitifs individuels. Il s'agit de prendre

*Email : pierre.willaimeduniv-lorraine.fr 
conscience du fait que nos croyances et connaissances ne dépendent pas seulement des éléments de preuves (ou evidences) en notre possession mais dépendent bien plus de nos interactions sociales, des groupes auxquels nous appartenons ou encore des institutions qui nous ont construits socialement. L'enquête sur la nature de la justification et la définition de la connaissance doit alors prendre en compte ces processus sociaux de génération de croyances. L'épistémologie sociale vise ainsi à réintégrer des aspects de nos vies épistémiques qui avaient été négligés par un certain goût, encouragé par tout un pan (internaliste) de la philosophie analytique contemporaine, pour l'introspection dans l'analyse de nos croyances et connaissances.

Le second but d'une épistémologie sociale est l'évaluation comparative de nos pratiques épistémiques ordinaires. Pour chaque étude de cas (qui constituent des application de l'épistémologie sociale), il nous faut identifier les actions à privilégier pour la quête de la connaissance. Cet objectif pratique contribue à une normativité revendiquée par Alvin Goldman et permet de considérer cette approche en philosophie de la connaissance non seulement comme une théorie pertinente conceptuellement, mais également comme un guide pour l'action.

Si les objectifs et les attentes d'une épistémologie sociale sont globalement partagés par ses défenseurs, il n'en est pas de même pour son architecture. La question de la nature précise d'une théorie sociale de la connaissance reste ouverte tout comme celle d'une méthodologie en vue de l'analyse des cas concrets. Les définitions de la connaissance pouvant satisfaire aux desiderata épistémiques d'une épistémologie sociale sont nombreuses et diverses. Doit-on examiner les pratiques épistémiques à la mesure de leur fiabilité (GOLDMAN 1994), du « travail » ou de l' « accomplissement » épistémique de l'agent (GRECO 2010) ou encore refuser la distinction entre la recherche d'éléments de preuve (évidentialisme) et l'analyse de la fiabilité des processus concourant à l'émergence de croyances pour combiner ces deux approches (COMESAÑA 2010) ? Dans ce qui suit, nous nous intéressons à l'analyse en terme de fiabilité des processus défendue par Alvin Goldman en présentant en quoi elle peut être considérée comme problématique pour le développement d'une épistémologie sociale.

\section{Fiabilité du processus ou fiabilité du résultat?}

\subsection{Qu'est-ce que le fiabilisme?}

La proposition d'épistémologie sociale la plus populaire est sans doute celle formulée par Alvin Goldman (1999). Celui-ci se base sur une théorie de la justification précise : le fiabilisme des processus. L'apparition du fiabilisme est antérieure à celle de l'épistémologie sociale. On en trouve des formulations chez Ramsey (1931), Unger (1968), Dretske (1971) ou encore Nozick (1981) mais c'est surtout dans un article de Goldman lui-même, « What is Justified Belief? » (1979), qu'il est présenté comme une alternative crédible à la définition classique de la connaissance pouvant éviter les cas à la Gettier ${ }^{1}$. Dans cet

1. Edmund Gettier est connu pour un célèbre article (en 1963) qui présente des contre-exemples à la définition classique de la connaissance comme croyance vraie justifiée. 
article, Goldman évalue différentes définitions fiabilistes de la connaissance et conclut sur la suivante :

Si la croyance de $S$ en $p$ à $t$ résulte d'un processus cognitif fiable, et si $S$ ne dispose pas de processus fiable ou conditionnellement fiable qui, si $S$ l'avait utilisé en plus du processus qu'il a effectivement utilisé, aurait eu pour résultat que $S$ ne croirait pas que $p$ à $t$, alors la croyance de $S$ en $p$ à $t$ est justifiée. (GOLDMAN 1979, p. 217)

Dans cette formulation du fiabilisme volontairement alambiquée pour répondre aux contre-exemples, nous pouvons noter deux choses : (1.) qu'il s'agit d'une théorie de la justification et (2.) que Goldman fait référence à des processus cognitifs fiables ou non. En effet, en 1979, Goldman développe une épistémologie individuelle, c'est-à-dire centrée sur l'agent épistémique et ses processus cognitifs «internes », décrite en particulier dans son livre Epistemology and cognition (1986). Le passage à une épistémologie sociale, que Goldman conçoit comme étant complémentaire avec le développement d'une épistémologie centrée uniquement sur l'individu, voit le fiabilisme s'élargir à des processus considérés comme "sociaux » et devenir principalement une théorie de la connaissance et accessoirement une théorie de la justification.

Cependant, l'épistémologie sociale de Goldman ne se base pas que sur le fiabilisme des processus. Celui-ci n'est que le premier pilier de l'architecture de la théorie goldmanienne qui s'articule avec un second fondement que Goldman appelle le «véritisme ». Par ce terme, il faut simplement entendre l'accent porté sur le lien indissociable entre la connaissance et la vérité. Ainsi, les pratiques sociales étudiées par Goldman sont évaluées par leur capacité à permettre aux agents épistémiques qui mettent en œuvre ces pratiques $\mathrm{d}^{\prime}$ atteindre la vérité (ce qui, pour Goldman revient à atteindre la connaissance ${ }^{2}$ ). Comme le dit Goldman :

[...] l'épistémologie sociale véritiste vise à évaluer les pratiques sociales par rapport à leurs résultats véritistes, ceux-ci pouvant être la connaissance, l'erreur ou l'ignorance. (GOLDMAN 1999, p. 87) ${ }^{3}$

Le véritisme n'est donc pas un simple parti-pris normatif ; il est le moyen par lequel le fiabilisme peut espérer s'appliquer à l'analyse de pratiques sociales épistémiques. Il s'agit d'une théorie causale qui nous demande d'identifier les causes de nos croyances; de les corréler avec de précédentes expériences (succès ou insuccès) et, finalement, de proportionner notre croyance au ratio d'accès à la vérité du processus en question. Par exemple, si je pose dix questions à un individu $x$ dans un certain contexte $c$ et que je peux vérifier, a posteriori, la vérité de sept d'entre elles, alors la fiabilité du processus de

2. Goldman assimile croyance vraie et connaissance, ce qui pose un certain nombre de problèmes, en particulier celui d'échouer à rendre compte d'une certaine "valeur » de la connaissance comparée à une croyance vraie (voir GOLDMAN et OLSSON (2009) pour une synthèse de ce problème et une tentative de réponse.)

3. «[...] veritistic social epistemology aims to evaluate social practices in terms of their veritistic outputs, where veritistic outputs include states like knowledge, error, and ignorance (GOLDMAN 1999, p. 87) ». 
génération de croyance « faire confiance à $x$ dans le contexte $c$ » est de 0.7 . Bien entendu, cet exemple doit être complexifié pour devenir pertinent et, même après raffinement, se heurte à de nombreuses difficultés.

Un des principaux problèmes du fiabilisme est d'identifier précisément quel processus de génération de croyances est fiable ou non. Chaque processus peut être décomposé en plusieurs mécanismes ou types de processus et il est très difficile de savoir exactement ce qui doit être fiable pour assurer la connaissance. Ce problème n'est pas propre à l'épistémologie sociale et concerne même plutôt les processus cognitifs de l'agent. CONEE et FELDMAN (1998) prennent l'exemple d'un individu, Smith, qui voit un érable depuis une fenêtre. Le processus le conduisant à penser qu'il y a un érable dans le jardin semble fiable. Cependant, de quel processus s'agit-il ? Il semble que ce qui est fiable dans ce cas est plutôt la chaîne d'événements qui va de la perception visuelle à la stimulation neuronale débouchant sur la croyance de Smith. Aucun des processus qui composent cette chaîne ne saurait être fiable par lui-même car ils dépendent les uns des autres pour conduire Smith à une croyance vraie. Or le fiabilisme cherche avant tout à identifier des processus clairement distincts, généraux, qui se répètent. Si ce sont des chaînes causales d'événements qui peuvent être qualifiées de fiables, alors chaque croyance dépend d'une chaîne différente (même si la différence est minime) et aucune généralisation ne peut être faite. Ce problème de la "généralité » est préoccupant pour l'épistémologie individuelle fiabiliste mais le versant social de l'épistémologie goldmanienne semble moins touché. Dans l'exemple qui suit, nous verrons pourquoi le problème de la généralité ne s'applique pas véritablement à l'épistémologie sociale. Au lieu de la sauver, cette relative immunité est en réalité un symptôme d'un problème plus large qui concerne l'épistémologie sociale fiabiliste.

\subsection{Le novice et les deux experts}

Un problème dorénavant classique en épistémologie sociale est celui des experts en désaccord. Goldman y a consacré un article (2001) et ce thème est aujourd'hui largement discuté, parfois comme un cas particulier du problème plus général tournant autour de la possibilité du désaccord rationnel. Ce cas concret reste suffisamment théorique, et classique, pour ne pas acter une séparation entre une épistémologie sociale appliquée et le reste de la philosophie de la connaissance pratiquant uniquement l'analyse conceptuelle.

Goldman formule le problème de la façon suivante : comment un novice qui est confronté à deux experts en désaccord peut-il trancher rationnellement? Cette question de la reconnaissance de l'expertise par celui qui ne peut précisément pas évaluer son contenu épistémique remonte au Charmide de Platon. L'épistémologie contemporaine doit pouvoir y répondre si elle veut éviter une forme de scepticisme social produit par une dépendance épistémique aveugle des novices. Même lorsqu'on conçoit ce type de connaissance comme dépendant majoritairement de la confiance du novice envers $l^{\prime}$ expert ${ }^{4}$, le cas d'un désaccord entre les experts force le novice à prendre position et à

\footnotetext{
4. C'est la position de HARDWIG (1985) qui soutient que la confiance implique forcément une dépen-
} 
justifier sa confiance plus grande dans l'un ou l'autre des experts.

Il semble nécessaire de préciser ce que l'on entend ici par « experts ». Pour Goldman, un expert dans un domaine donné est quelqu'un qui a plus de croyances dans des propositions vraies et/ou peu de croyances dans des propositions fausses par rapport aux croyances (relatives au domaine) de la majorité des autres individus (GOLDMAN 2001, p. 90-91). Là encore, l'analyse de Goldman repose fortement sur le pilier du véritisme. Goldman affine son analyse en distinguant une fausse forme d'expertise, celle de l'expert « réputationnel » qui est considéré comme un expert par une communauté sans que ce soit véritablement le cas. De plus, l'expertise ne doit pas se cantonner à la possession d'un ensemble d'informations justes et précises. Pour parler de connaissance, l'expert doit être capable de déployer son savoir pour répondre à de nouvelles questions de son domaine de compétence. Ainsi, l'expert se définit également par son savoir-faire et ses compétences techniques.

Goldman analyse rapidement trois réponses classiques au problème du novice et des deux experts. La première consiste à organiser un débat entre les deux experts pour permettre au novice de prendre parti. Si cette proposition a le mérite de permettre l'échange d'arguments, le problème de la dépendance épistémique décrit par HARDWIG (1985) refait surface. Comment, en effet, un novice peut-il comprendre et évaluer des arguments d'un domaine de connaissance qui lui est totalement étranger? Comme le dit Goldman, le novice ne pourra pas trancher parmi des propositions qui seront pour lui ésotériques. Pire encore, écouter un débat sans pouvoir en saisir le contenu peut engendre des biais ; l'orateur le plus charismatique ou le plus pédagogue peut l'emporter alors même que ses arguments sont fallacieux. La seconde possibilité est de faire appel à des méta-experts qui seront chargés d'évaluer les premiers experts. Ces méta-experts peuvent en effet comprendre les arguments en jeu et trancher. Cependant, cette proposition ne fait que repousser le problème d'un niveau; comment le novice peut-il trancher si les méta-experts ne s'accordent pas? Convoquer à nouveau d'autres experts nous conduit à une régression à l'infini. La troisième solution présentée par Goldman est de demander l'avis de la communauté scientifique sur la question. Dans un débat entre deux personnes, une position minoritaire et habituellement déconsidérée peut apparaître comme pertinente par le choix des experts ; l'avis de la majorité des experts du domaine pourrait éviter ce biais. Cependant, Goldman note que la majorité n'a pas toujours raison et peut soutenir des positions pour une multitude de raison dont une fraction seulement sont pertinentes épistémiquement. De plus, suivre toujours l'avis de la majorité conduirait à une forme de conformisme intellectuel négatif pour la quête de la connaissance.

$\mathrm{Au}$ final, Goldman introduit une nouvelle solution à ce problème du novice et des deux experts. Sa proposition est un exemple type d'application du fiabilisme des processus à une étude de cas d'épistémologie sociale. Goldman propose d'enquêter sur les experts afin d'établir un historique de leurs prises de positions antérieures («Past Track Record»). Son idée principale est que certaines de ces prises de décision auparavant inaccessibles au novice car ésotériques lui sont maintenant compréhensibles. Il

dance au moins partiellement aveugle du novice. 
s'agit de thèses susceptibles d'être contredites par des évènements ou des questions sur lesquels un consensus scientifique s'est établi par la suite. Ainsi, on peut dire qu'un expert qui soutenait une position aujourd'hui universellement considérée comme fausse s'est trompé à l'époque. Le recul dû à la distance temporelle rend exotériques un certain nombre d'affirmations qui étaient ésotériques.

La solution de Goldman est intéressante mais souffre de nombreuses limites. Elle est restreinte à l'évaluation de propositions prédictives ou, en tout cas, susceptibles d'être invalidées; ce qui n'est pas le cas d'un grand nombre de thèses en sciences "dures » comme en sciences humaines. Certes, des consensus se forment même dans des domaines non prédictifs ou qui n'établissent pas d'arguments «massues » contredisant définitivement une théorie. Mais la réponse de Goldman à la troisième solution classique se retourne alors contre lui : si suivre l'avis majoritaire n'est pas toujours pertinent pour le novice (par risque d'un certain conformisme intellectuel qui entraverait le progrès, par peur des effets de mode, ...), pourquoi le serait-elle pour évaluer les "états de service» des experts? Nous pouvons également être face à des cas de chance ou malchance épistémique : un expert pourrait avoir statistiquement plutôt raison mais souvent sans véritable base épistémique (il ne fait que suivre l'avis de quelqu'un d'autre sans aucun recul ; il «sent » l'évolution des positions ; ...). Inversement un expert très compétent peut avoir tort en dépit du bon sens; ses processus de formation de croyances sont fiables, il est vertueux intellectuellement et pourtant se trompe, par malchance.

Outre les limites «techniques » de la solution fiabiliste de Goldman, ce sont ses présupposés qui posent question. L'approche fiabiliste basée sur l'évaluation des prises de positions antérieures des experts est très différente des trois solutions classiques. Ces dernières tentent $d$ 'atteindre les raisons pour lesquelles les experts défendent leur point de vue. Elles tentent de remonter la chaîne des justifications pour accéder au contenu épistémique de l'énoncé. À l'inverse, la quatrième solution ne cherche plus les causes de la croyance des experts. Elle n'évalue pas le processus par lequel les experts en sont venus à soutenir leur position. Elle évalue la propension des experts à soutenir des propositions qui s'avèrent vraies. Peu importe le cheminement intellectuel des experts et leurs interactions, ce qui compte au final c'est leur résultat compris en terme de ratio entre erreurs, connaissances et ignorance comme le veut le véritisme goldmanien.

\section{3 Épistémologie sociale naturalisée}

Il nous faut donc séparer deux conséquences de la proposition de résolution du dilemme du novice. Premièrement, cette solution est limitée dans son usage comme dans son pouvoir explicatif. À ce titre, elle se restreint à un certain type de connaissances et elle ne s'applique qu'à des domaines pour lesquels nous disposons de données archivées et de prévisions à court terme. La seconde conséquence de la proposition goldmanienne est un changement de nature de l'enquête épistémique. L'analyse conceptuelle en philosophie de la connaissance se demande comment expliquer et définir des notions telles que connaissance ou justification. La suggestion fiabiliste de réponse au problème $\mathrm{du}$ novice et des experts se demande comment maximiser les chances du novice de soutenir une croyance vraie. Une définition de la justification est donnée (le novice est justifié à 
croire la position de celui qui s'est statistiquement le moins trompé dans le passé), mais c'est une définition ad hoc qui ne cherche pas véritablement à saisir ce que renferme le concept de justification tel que nous l'utilisons dans notre vie quotidienne.

L'exemple du novice et des experts met en scène le fonctionnement et l'architecture de l'épistémologie sociale goldmanienne. Nous pouvons nous rendre compte du glissement qu'elle opère au sein de la philosophie de la connaissance. Le but ultime de Goldman étant d'atteindre la vérité, il redéfinit les termes classiques de l'épistémologie ; la justification du novice, la confiance ou l'autorité d'un individu sont évalués sous un seul critère : celui de la propension au vrai. L'expert qui ne se trompe que $10 \%$ du temps est digne de confiance, peu importe sa manière de travailler, son attitude ou son aura dans la communauté. En définitive, Goldman semble penser que tout accès à une croyance vraie présuppose un processus causal fiable alors que seul l'inverse, c'est-à-dire que tout processus causal fiable débouche sur une croyance vraie, pourrait être acceptable (et encore, ce serait ne pas prendre en compte la possibilité d'insuccès épistémiques dû à la malchance).

Le traitement de l'étude de cas en question par Goldman contraste avec sa position concernant des relations entre philosophie de la connaissance traditionnelle et épistémologie sociale. Sur cette question, Goldman défend une approche qu'il qualifie de préservationniste dans «Why Social Epistemology Is Real Epistemology », un article qui, comme son nom l'indique, vise précisément à rapprocher l'épistémologie sociale du reste de l'épistémologie. Le préservationnisme conçoit l'épistémologie sociale comme compatible avec le développement de l'épistémologie traditionnelle centrée sur l'individu. Le versant social de l'épistémologie se contenterait d'introduire de nouveaux objets d'études tels que les groupes ou les institutions, mais n'invaliderait en aucun cas le projet général de la philosophie de la connaissance. Goldman veut en fait se distinguer d'une position qu'il décrit comme révisionniste et qu'il attribue à la sociologie de la connaissance et aux philosophies qui ont abandonné l'analyse de la connaissance en termes normatifs pour une approche descriptive des pratiques épistémiques.

Le fiabilisme semble entrer en contradiction avec ce projet préservationniste. Certes, il est possible de contribuer au développement d'une épistémologie traditionnelle tout en travaillant dans une épistémologie sociale fiabiliste. Cependant, les deux approches sont difficilement conciliables. Le fiabilisme, combiné au véritisme, cherche la justification dans la propension à atteindre la vérité et ne peut pas analyser spécifiquement les processus tels que la confiance ou l'autorité épistémique. Goldman propose une forme de réductionnisme des concepts d'épistémologie à leur résultat véritistique. Ce n'est plus le mode de formation des croyances qui intéresse l'épistémologue social, c'est le résultat de ce mode. Or, n'est-ce pas une conséquence principale des cas à la Gettier d'établir que ce n'est pas le résultat qui compte, mais la manière dont on y arrive? En effet, entretenir une croyance vraie accompagnée de justification ne suffit pas pour la connaissance car le processus épistémique suivi peut ne pas être pertinent pour la connaissance en question.

La position de Goldman semble bien peu préservationniste vis-à-vis de la philosophie de la connaissance traditionnelle. Certes Goldman ne souhaite pas attribuer des états mentaux aux agents collectifs tels que les groupes comme le veulent des partisans de l'approche expansionniste en épistémologie sociale. Toutefois, il redéfinit les notions 
centrales de l'épistémologie en termes de probabilité statistique de former une croyance vraie. Cette forme de naturalisation quelque peu bayesienne ${ }^{5}$ de l'épistémologie n'a plus rien à voir avec l'enquête épistémique telle qu'elle était menée jusqu'alors. Deux possibilités s'offrent à nous ; nous pouvons embrasser ce nouveau projet et revendiquer une nouvelle épistémologie qui permettrait un traitement bayesien des études de cas et une modélisation des interactions épistémiques. L'apport de ces nouvelles méthodes en philosophie serait contrebalancé par l'abandon des anciens débats conceptuels et d'une redéfinition de nos concepts. Nous pouvons aussi choisir de développer une épistémologie sociale qui n'adopterait pas la méthode naturaliste proposée par le fiabilisme tel que le conçoit Goldman. L'épistémologie des vertus peut être considérée comme une candidate pour une telle épistémologie sociale ; ce qui suit présente cette approche en soulignant une difficulté.

\section{Un problème pour une épistémologie sociale arétique}

\section{1 Épistémologie sociale et vertus intellectuelles}

L'épistémologie sociale n'est pas aussi unifiée que la présentation de Goldman pouvait le suggérer. Il est préférable de parler d'épistémologies sociales plutôt que d'une épistémologie sociale. Certes, une telle approche doit adopter un certain nombre de thèses pour pouvoir prétendre échapper à l'individualisme de l'épistémologie classique. En particulier, on voit mal une épistémologie sociale se revendiquer internaliste. La prise en compte du caractère social de la connaissance semble ne pas pouvoir accompagner une théorie qui ne ferait qu'étudier les états mentaux de l'agent conçus de manière autonome. Certes, peu d'internalistes soutiendraient l'idée radicale selon laquelle des facteurs habituellement caractérisés comme «sociaux » ne joueraient aucun rôle dans la formation de croyances. Pour autant, même en reconnaissant une influence contextuelle, l'internalisme conçoit généralement le sujet comme pratiquant une évaluation introspective de ses raisons de croire ; ce point de vue est difficilement compatible avec le mode de fonctionnement des vertus épistémiques, qui résulte d'une habitude prise par l'agent qui n'est donc pas le juge ultime validant ses processus cognitifs. Dans une conception arétique de la connaissance, l'agent n'agit pas directement sur ses raisons de croire mais peut modifier son comportement, ce qui aura pour effet, à terme, de modifier ses dispositions intellectuelles et donc ses raisons épistémiques. De même que le refus d'un internalisme fort, celui de réductionnisme ${ }^{6}$ dans l'analyse du témoignage apparaît comme un prérequis à une approche sociale de la connaissance. Mais en dehors d'un externalisme et d'un anti-réductionnisme assez généraux, la fondation d'une épistémologie sociale peut s'accommoder de plusieurs définitions de la connaissance et de la

5. L'inférence bayesienne désigne de manière large les méthodes qui permettent de déduire la probabilité d'un événement à partir de celles d'autres événements antérieurs du même type. Le traitement du problème du novice et des experts par Goldman permet précisément ce type d'analyse statistique.

6. Le réductionnisme conçoit la connaissance issue du témoignage comme dérivée des connaissances perceptives. Ainsi, le témoignage produirait des connaissances de second zone dont la solidité n'égalerait pas celles produites par l'individu seul. 
justification.

Une théorie candidate et relativement populaire aujourd'hui est l'épistémologie des vertus. Selon cette dernière, nous gagnerons à analyser la connaissance comme résultante de l'exercice de vertus intellectuelles. L'épistémologie des vertus a deux avantages principaux pour servir de socle à une épistémologie sociale :

- Il s'agit d'une théorie centrée sur l'individu mais pour laquelle le contexte joue un rôle très important. Après une théorie fiabiliste qui portait uniquement sur les processus de formation de croyance, le recentrage sur l'individu est bienvenu. Le danger était d'oublier par la même occasion les critiques portées contre la philosophie de la connaissance classique qui place trop souvent le sujet comme maître et possesseur de ses croyances. La notion spécifique de "vertu » échappe par définition à un contrôle épistémique de la part de l'agent. Dans le cadre d'une épistémologie des vertus, le sujet est remplacé par l'agent. Alors que le sujet pouvait librement agir sur ses croyances (volontarisme doxastique), l'agent est responsable de son caractère vertueux ou vicieux et ne peut en changer facilement; la vertu étant une disposition, acquise par la répétition d'actions, à se comporter d'une certaine manière, il faut du temps pour en changer.

- La notion de vertu contient en elle-même la prise en compte de la formation sociale des croyances. À l'inverse, le fiabilisme peut être centré uniquement sur l'individu. Dans la première version du fiabilisme développée par Goldman dans Epistemology and cognition (1986), il ne s'agissait que de rendre compte des processus cognitifs internes à l'individu. Goldman voulait dresser un pont entre les sciences cognitives (et en particulier la psychologie cognitive) et l'épistémologie. L'épistémologie des vertus n'est pas aussi lâche conceptuellement et ne peut désigner une théorie internaliste de la justification.

\subsection{Explication versus prescription}

Dans le passage d'une épistémologie fiabiliste à une épistémologie des vertus, il y a tout de même une certaine perte. Le fiabilisme pouvait s'acquitter des deux rôles de l'épistémologie sociale, à savoir le développement d'une théorie de la connaissance sociale et un objectif prescriptif d'identification des bonnes pratiques épistémiques. L'épistémologie des vertus permet de fournir une explication normative de la génération des croyances d'une manière quasiment systématique. Par contre, elle semble échouer à fournir un guide pour l'action. Dire à une personne « sois tempérant! » ne lui indique en effet pas précisément quels comportements adopter. Aristote note dans l'Éthique à Nicomaque (II, 9, 1109a-1109b) que la recherche du juste milieu entre deux excès qui est un guide pratique pour atteindre la vertu n'est pas chose facile et nécessite un travail individuel ${ }^{7}$. Bien qu'à visée pratique, l'épistémologie des vertus ne veut pas

7. «Quant à dire jusqu'à quel point et dans quelle mesure la déviation est répréhensible, c'est là une chose qu'il est malaisé de déterminer rationnellement, comme c'est d'ailleurs le cas pour tous les objets perçus par les sens : de telles précisions sont du domaine de l'individuel. Éthique à Nicomaque, trad. Tricot $1109 \mathrm{~b} »$ 
et ne peut pas fournir des recommandations éthiques précises. La latitude nécessaire à l'appréciation de la vertu empêche alors de développer une épistémologie sociale arétique qui évaluerait des pratiques sociales d'un point de vue global. Au mieux, une telle épistémologie pourrait rendre compte a posteriori de cas précis et bien documentés sans pouvoir les généraliser.

En échouant à prescrire efficacement, l'épistémologie des vertus ne remplit pas un rôle fondamental de la philosophie de la connaissance, à savoir permettre aux individus de reconnaître les sources d'informations fiables. CRAIG (1990) décrit précisément ce rôle de l'épistémologie dans son ouvrage Knowledge and the state of nature : an essay in conceptual synthesis. Selon Craig, toute communauté pratique et doit pratiquer une évaluation des sources d'informations. Le concept de connaissance sert précisément à marquer et à identifier une source d'information fiable susceptible d'être utilisée par les membres de la communauté. Le rôle de l'épistémologie est alors non seulement de comprendre ce mécanisme naturel par lequel les membres d'une communauté font confiance à certains d'entre eux, mais également de pouvoir leur indiquer des critères et des recommandations pour favoriser la circulation des informations. C'est précisément ce second rôle qui est problématique pour une épistémologie des vertus trop théorique.

\subsection{L’analyse de modèles épistémiques}

Pour pouvoir couvrir tous les rôles afférents à une épistémologie sociale, l'épistémologie des vertus devrait s'enrichir d'une capacité à guider l'individu, le groupe ou encore l'institution. En fait, c'est l'application de cette position théorique qui pose problème. Il faudrait accompagner l'analyse arétique par des analyses prescriptives de cas concrets. Pour cela, il faut développer une certaine méthodologie. Pour chaque cas concret étudié, l'épistémologie sociale arétique doit, en plus de fournir une explication pertinente de la situation dans son cadre théorique, proposer des solutions possibles au problème posé. Il serait envisageable, par exemple, d'analyser le problème du novice et des deux experts avec la terminologie et le cadre conceptuel de l'épistémologie des vertus. Une telle analyse pourrait suggérer d'autres solutions au dilemme du novice.

Nous pouvons comprendre cet aspect prescriptif de l'épistémologie sociale comme étant une analyse de différents modèles épistémiques, un modèle épistémique étant un ensemble de comportements, pratiques ou idées implicites ou explicites en vue de la connaissance. Chaque entité sociale (groupe, institution, ...) est régie par une certaine conception de la connaissance revendiquée (modèle épistémique explicite qui regroupe les comportements attendus de la part des membres de l'entité sociale) mais aussi implicite (qui regroupe les comportements réels, qui peuvent différer de ceux attendus).

Les communautés épistémiques en ligne se prêtent relativement facilement à ce type d'analyse. Ainsi, l'ensemble des règles qui régissent le fonctionnement de l'encyclopédie libre Wikipédia donne une idée assez précise de la conception de la connaissance qui y est sous-entendue. Nous pourrions qualifier ce modèle comme étant une vision fiabiliste de la connaissance car tout est fait pour ne pas prendre en considération l'agent, le contributeur, pour se concentrer au contraire sur le contenu, sur les modifications apportées à l'encyclopédie. Peu importe qui écrit, c'est le respect d'un certain processus (respect des 
règles, discussion, auto-correction, ...) qui permet à une modification d'être considérée comme fiable par la communauté. Cependant, lorsqu'on s'intéresse aux pratiques réelles des membres de cette communauté épistémique, il apparaît assez clairement que le rôle de l'agent et de son appréciation sociale est central dans le fonctionnement de l'encyclopédie. L'utilisation, en particulier, de l'historique des contributions pour évaluer la compétence d'un individu, ses possibles biais et intérêts contredit l'idée d'une construction de l'encyclopédie par l'analyse seule du contenu et de la forme des contributions. L'importance accordée implicitement à une certaine forme de réputation permettant la confiance entre les contributeurs est primordiales pour saisir le fonctionnement de cette encyclopédie. Au final, une explication des mêmes comportements par le cadre théorique de l'épistémologie des vertus apparaît pertinente. Le fait de considérer les actions des contributeurs comme étant le produit d'une certaine forme de dispositions intellectuelles permet alors de formuler des propositions d'améliorations de ce projet d'encyclopédie libre et ouverte ${ }^{8}$.

\section{Références}

COMESAÑA, Juan (2010), « Evidentialist Reliabilism », in : Noûs 44.4, p. 571-600.

CONEE, Earl et Richard FeldMAN (1998), "The Generality Problem for Reliabilism », in : Philosophical Studies : An International Journal for Philosophy in the Analytic Tradition 89.1, p. 1-29.

CRAIG, Edward (1990), Knowledge and the state of nature : an essay in conceptual synthesis, Oxford : New York : Clarendon Press; Oxford University Press.

Goldman, Alvin I. (1979), "What is Justified Belief? ", in : Justification and Knowledge, sous la dir. de George Sotiros PAPPAS, t. 17, Philosophical Studies Series in Philosophy, Springer Netherlands, p. 1-23, trad. par Emmanuelle GLON sous le titre «Qu'est-ce qu'une croyance justifiée? », in : DUTANT, Julien et Pascal ENGEL, Philosophie de la connaissance : croyance, connaissance, justification (Paris : J. Vrin, 2005).

- (1986), Epistemology and cognition, Cambridge, Mass. : Harvard University Press.

- (1994), «Naturalistic Epistemology and Reliabilism », in : Midwest Studies In Philosophy 19.1 , p. 301-320.

- (1999), Knowledge in a Social World, Oxford; New York: Clarendon Press ; Oxford University Press.

- (2001), «Experts : which ones should you trust?», in : Philosophy and Phenomenological Research 63.1, p. 85-110.

- (2010), «Why Social Epistemology Is Real Epistemology », in : Social Epistemology, sous la dir. d'Adrian HADDOCK, Alan MILlAR et Duncan PRITCHARD, Oxford University Press, p. 1-28.

GOLDMAN, Alvin I. et Erik J. OlssOn (2009), «Reliabilism and the Value of Knowledge», in : Epistemic value, p. 19-41.

GRECO, John (2010), Achieving knowledge : a virtue-theoretic account of epistemic normativity, Cambridge; New York : Cambridge University Press, 205 p.

8. Voir WiLlaime (2015) pour une analyse plus détaillée de la question. 
HARDWIG, John (1985), «Epistemic Dependence », in : The Journal of Philosophy 82.7, p. 335.

WILlAIME, Pierre (2015), «Une analyse épistémologique de l'expertise dans Wikipédia », in : Wikipedia : objet scientifique non identifié, sous la dir. de Lionel BARBE, Louise MERZEAU et Valérie SCHAFER, Intelligences numériques, Presses universitaires de Paris Ouest. 